\title{
VI. Nutritional Care and Related Studies
}

\author{
ELAINE POTTS, CHARLES T. L. HUANG, AND BUFORD L. NICHOLS \\ Department of Pediatrics, Baylor College of Medicine and Clinical Research Center, Texas Children's Hospital, \\ Houston, Texas USA
}

\section{PREPARATION AND STERILIZATION OF FOODS AND SUPPLIES}

All foods and formula used for this child thus far have been commercially available as "sterile preparation." These have been purchased in quantity lots from single preparations. The formula and canned foods are not resterilized but samples of each lot are always cultured for microbial contaminants. Those lots which show contamination are discarded. To prepare the exterior surface of bottles and jars for sterilization, labels and glue are removed and the containers are carefully checked for chipped glass or damaged cans. They are then placed in cardboard boxes in two layers with a single layer of paper between. Nipples, along with plastic bowls and utensils, are placed loosely in open paper bags. Containers of food, formula, water, and supplies for specific time periods are put into cylinders, sealed with Mylar, and sterilized by placing them in ethylene oxide gas at $140^{\circ}$ for $4 \mathrm{hr}$, then room aerating for 7 days (or for $8 \mathrm{hr}$ in an aerator) before putting them into the isolator. Steam autoclaving of nipples is optional. Vitamins and supplements are sterilized by passing through a Millipore filter of $0.22 / \mathrm{nm}$ porosity.

\section{DIET}

At birth the infant was placed on Enfamil formula, $15 \mathrm{cal} / \mathrm{oz}$, which was soon increased to $20 \mathrm{cal} / \mathrm{oz}$ to satisfy hunger. Distilled water was used initially for drinking. At 1 month of age he still seemed to be hungry so fruits and vegetables were introduced and 1 week later strained meat and egg yolk were introduced. New foods were always introduced in restricted variety in order to identify any to which he might be allergic or intolerant. However, the eating habits have been those of a normal child with few problems.

A variety of foods was gradually introduced into the diet at about 3 months of age. Although his dislikes have been very limited, liver has been completely rejected and corn and sweet potatoes are not favorites with him. All attempts to incorporate liver into the diet have been unsuccessful. Although egg yolks have been included in the diet earlier it became evident that these tended to be contaminated so they were discontinued. Teething biscuits and many types of cookies also could not be included because they showed microbial contamination in spite of the use of various techniques for cooking and sterilizing them. It soon became clear that most foods were microbiologically safe except for those with the highest fat contents, including certain canned meat products.

Junior foods were introduced at about 9 months of age, in order to give foods with a coarser texture. As with any normal child, the patient contemplated these changes suspiciously but soon accepted them. The toddler foods, in limited amounts, were gradually added at 12-14 months of age. These were introduced in order to give him the opportunity to touch foods and to stimulate his interest in chewing. He was apprehensive about picking up foods and putting them in his mouth. Neverthe- less, he gradually accepted them and by 18 months of age he was feeding himself and drinking from a cup.

The diet at 3 years of age contained a variety of canned junior and toddler foods, chopped fruits and desserts. The daily caloric intake was $1,450-1,500$, including $65-70 \mathrm{~g}$ protein (20\%), 50$55 \mathrm{~g}$ fat $(35 \%)$, and $165-175 \mathrm{~g}$ carbohydrate. The caloric intake was kept at reasonably low levels, just sufficient for normal growth and maintenance. The diet was always a little high in protein. At 3 years he was still on formula with added iron, his main source of iron. A multiple vitamin supplement was given by mouth in a dosage of 0.6 cc daily. Tap water was sterilized for drinking.

The cholesterol intake had always been low and was less than $150 \mathrm{mg}$ daily, since the egg products were eliminated and he refused liver. This low cholesterol intake led to interesting studies as described below.

Dietary problems for the future include the introduction of table foods. For this, special methods of sterilization will be needed. More fiber in the diet would also be desirable for dental health and to increase the bile acid secretion. It would also be helpful to discover whether fat is being adequately absorbed and to determine whether fat-soluble vitamins are being utilized.

\section{NUTRITIONAL PROBLEMS AND RELATED STUDIES}

\section{LOSS OF HAIR AND SKIN CHANGES}

When the patient was 3 months of age he showed scalp changes, including loss of hair and rough skin. This led to the question of whether he was getting enough folic, biotic, and linoleic acid for his particular needs. The synthesis of folic acid may occur through the action of microorganisms in the intestinal tract, and biotic is shown to be necessary for the synthesis. In this case, where the intestinal flora was absent, there was a question as to whether a normal intake of minerals and vitamins in the food and formula was sufficient. It was also not known whether $D V$ was properly absorbing and utilizing the amounts given. He had not been on vitamin supplements at that time and the formula was not iron fortified, nor did it contain biotic other than the amount in milk which is ordinarily sufficient for growth in normal children. The patient had been on gentamicin and later on kanamycin in an attempt to eradicate a $S$. epidermidis contamination, but the gentamicin had been discontinued a month before and the kanamycin 2 weeks before the scalp changes occurred. The child was placed on an iron fortified formula and vitamins were given. Vitamin C (1 cc Berroca C) was given daily by mouth and $5 \mathrm{mg}$ folic acid and $500 \mu \mathrm{g} B_{12}$ intramuscularly once a month. The scalp problem soon cleared up. The Berroca $C$, a preparation for intravenous use, was selected for its sterility but $D V$ disliked it intensely. Therefore, it was discontinued and he was placed on a multivitamin preparation (Vi-Penta no. 3) which contained adequate vitamin C. At this time vitamin assays were done on urine and serum samples (69). The results, shown in Table 1 show that he was absorbing 
Table 1. Vitamin assays of gnotobiotic child at age 6 months (69)

\begin{tabular}{|c|c|c|c|}
\hline Vitamin & Assay 1 & Assay 2 & $\begin{array}{c}\text { Normal range, } \\
0-5 \text { mo }\end{array}$ \\
\hline Vitamin $A, \mu g / 100 \mathrm{ml}$ & & 78.0 & 20.0 or $>$ \\
\hline Vitamin $\mathrm{C}, \mathrm{mg} / 100 \mathrm{ml}$ & 0.4 & 2.5 & 0.3 or $>$ \\
\hline $\begin{array}{l}\text { Thiamine/creatinine, } \\
\mu \mathrm{g} / \mathrm{g}\end{array}$ & $12,510.0$ & $15,110.0$ & 176.0 or $>$ \\
\hline $\begin{array}{l}\text { Riboflavin/creatinine, } \\
\mu \mathrm{g} / \mathrm{g}\end{array}$ & $13,723.0$ & $20,161.0$ & 50.0 or $>$ \\
\hline
\end{tabular}

vitamins normally according to established ranges for normal infants up to 5 months of age (51). The vitamin supplements undoubtedly account for the high levels of thiamine/creatinine and riboflavin/creatinine found in the urine.

Another episode of hair loss and dry skin occurred at 15 months. He had been home for approximately 6 weeks and had not been given his vitamin supplements. Vitamins were given again and subsequently the scalp problems improved. There was, however, no proof that the scalp problems were related to a vitamin deficiency. In view of the possibility that the use of distilled water had resulted in a lack of trace minerals, a sample of hair was analyzed. No deficiency was detected, but it was decided to introduce sterile tap water at that time.

\section{EFFECT OF LOW CHOLESTEROL DIET ON MYELINIZATION}

Bile acids and neutral steroids normally excreted by the liver into the intestinal tract are subjected to further metabolic change by the action of the intestinal bacteria. They are largely reabsorbed so that the amount found in the stool is less than $5-10 \%$ of that excreted by the liver daily. The total bile acid pool will turn over twice during a meal, thus allowing rapid recycling and reutilization of bile acids. Cholesterol comes from the diet and also is synthesized in the body. In general, a low cholesterol diet is associated with an increased synthesis of cholesterol to make up for the dietary deficit. There has, however, been controversy in the literature about the treatment of children with congenital high blood cholesterol by low cholesterol diets. Some have argued that this would not allow adequate myelinization and central nervous system development and have suggested that low cholesterol diet not be used in infancy. Obviously $D V$ is a child who has been on extremely low cholesterol intakes from birth, yet there is no evidence of malfunction of the nervous system or delay in myelinization. This is a very important observation with therapeutic implication for children with congenital hypercholesterolemia.

\section{COMPARISON OF FECAL BILE ACIDS AND NEUTRAL STEROIDS WITH THOSE OF CONTROL CHILDREN}

Normally, bile acids synthesized and secreted by the liver are conjugated with glycine or taurine. The bacterial modification (deamidation and dehydroxylation) is a product of anaerobic bacteria. Unpublished studies done in this laboratory show that children under 1.5 years excrete more primary bile acids in the stool, $28.2 \pm 6.2 \%$ than adults, who excrete only $5 \%$, the remaining bile acids appearing in secondary forms. This is probably due to slower transit time in adults where the greater intestinal length provides more bacterial interaction with sterols. Figure 1 shows the pattern of the acids for $D V$ at the age of 2 years (when he did have some anaerobic bacteria in the fecal flora), compared with control children under 1.5 years of age. Although $D V$ had a number of secondary bile acids, he was excreting a larger percentage of primary bile acids than the control subjects, approximately $70 \%$. This was mostly in the form of deconjugated cholic and chenodeoxycholic acids. This indicates that $D V$ had a reduced activity of bacterial flora in terms of conversion of the primary bile acids to secondary products.

The other fraction of fecal sterol lipid is that containing cho- lesterol and its metabolic products. In Figure 2, the patterns of cholesterol and other neutral lipids are illustrated along with the plant sterols which are a function of the dietary plant intake. The animal sterols can be synthesized in the body as well as derived from dietary sources. In adults about $25 \%$ of the total endogenous neutral sterols are excreted as cholesterol. The rest are excreted as coprostanol which has had a bacterial modification in the $\Delta^{5}$ double bond, resulting in a saturated chemical form. In children at 4 years of age, the pattern is not unlike the adult, but in children under 1.5 years of age $<95 \%$ of the cholesterol is still in the unmodified form, i. e., the sterol nucleus has not undergone saturation in the $\Delta^{s}$ double bond. This results in a smaller amount of fecal coprostanol. $D V$, at age 2 years, excreted $99.4 \%$ of his neutral lipids as cholesterol with just a trace of coprostanol, whereas control subjects of 1.5 years or less showed $<95 \%$ cholesterol with about $5 \%$ coprostanol. We have had an opportunity to see the same kind of pattern that $D V$ presented in patients with cholera-like diarrhea where the sterols pass through the intestine so rapidly that there is no time for bacterial modification. In our patient it was the modification of his bacterial flora which affected the intestinal metabolism of cholesterol. The exact opposite is seen in some patients with chronic, non-

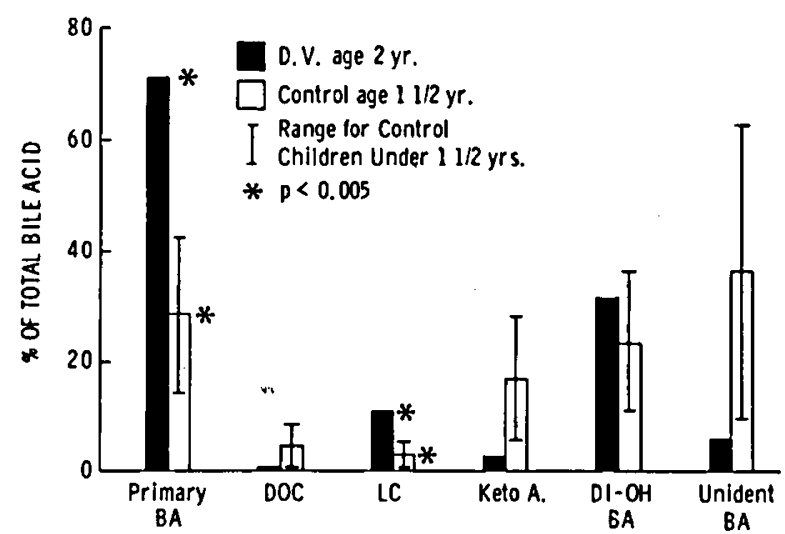

Fig. 1. Fecal bile acid profile of gnotobiotic child at age 2 years. Primary BA: sum of cholic and chenodeoxycholic acids; DOC: deoxycholic acid; LC: lithocholic acid; Keto A: keto-hydroxy bile acids; DIOH BA: sum of dihydroxy bile acids including chenodeoxycholic, deoxycholic, ursodeoxycholic, and isodeoxycholic acids. Unident BA: unidentified bile acids.

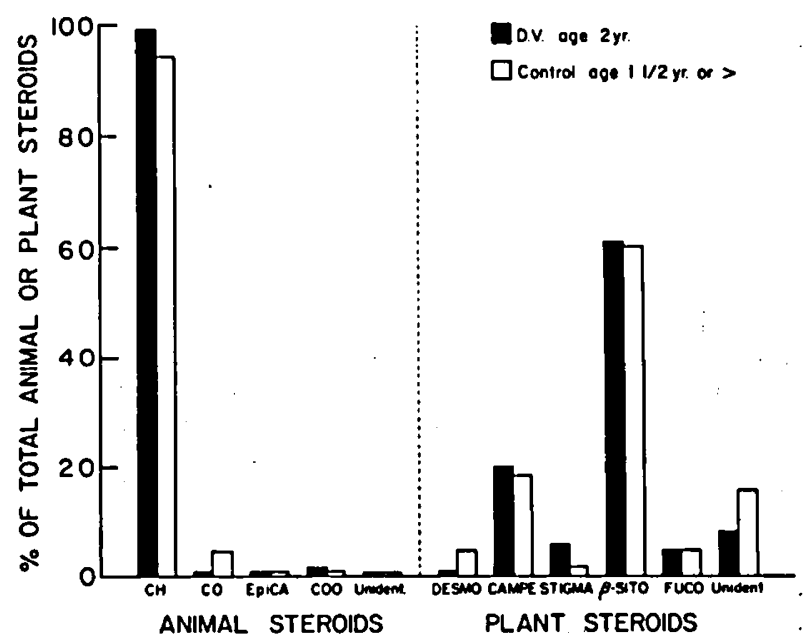

Fig 2. Fecal neutral steroid profile of gnotobiotic child at age 2 years. $\mathrm{CH}$ : cholesterol; $\mathrm{CO}$ : coprostanol; EpiCA: epicholestanol; $\mathrm{COO}$ : coprostanone; Unident.: unidentified; DESMO: desmonterol; CAMPE: campesterol; STIGMA: stigmasterol; $\beta$-SITO: $\beta$-sitosterol; FUCO: fucosterol. 
specific diarrheal syndrome. These are children with a massive overgrowth of anaerobic bacteria in the upper small bowel. They excrete relatively small amounts of unchanged neutral lipids and small amounts of primary bile acids in the feces because of increased bacterial action.

It would be interesting to know to what extent a modified bacterial flora affects cholesterol or sterol balance. In addition to the well known relationship between diet and blood cholesterol levels, other factors may come into play. Normal people in differing countries have a wide range of intestinal function. The
D-xylose absorption and fat absorption tests have different ranges of normal values. It would be interesting to know whether increases in bacterial flora are playing a role in the lower blood cholesterols seen in patients in developing areas where sanitation is, in general, very much poorer than in North America. $D V$, for instance, represents the extreme of sanitation. Studies by Kellogg (27) indicate that in rats in a germ-free environment, hepatic cholesterol and blood cholesterol are increased. The serum cholesterol value in $D V$ is low, but this undoubtedly is a reflection of his extremely low cholesterol intake.

\title{
VII. Mental, Psychomotor, and Psychosocial Development
}

\author{
BARRY MOLISH, MARY MURPHY, AND MURDINA DESMOND
}

The Department of Pediatrics, Baylor College of Medicine, The Leopold Meyer Center for Developmental Pediatrics, Texas Children's Hospital, Houston, Texas, USA

The following data are based upon observation of $D V$ 's behavior as well as on the results of standardized developmental schedules and intelligence scales. They reflect his ability and achievements as accurately as possible within the limitations imposed by his unique environment on the testing procedures, such as the necessity for sterilization and modification of some of the test items.

\section{INITIAL EVALUATION}

The initial evaluation was on March 15, 1972 when he was 5 months, 24 days of age. The Bayley Scales of Infant Development (6) were selected to obtain a baseline measure for reference in assessing his future longitudinal course of development. The findings were: Mental Scale Index: 116 (6.5-7.5 months); Psychomotor Scale Index: 112 (6.5-7.5 months).

On the Mental Scale Index this child was approximately 1 month above his age level. This was probably an underestimate because of four items scored as "omitted" because of difficulty in administration. Basal Age (level of consistent success of 10 consecutive passes) was established as 5.7 months. Ceiling Age (highest level of success before 10 consecutive failures) was 8.9 months. Items passed included: transfers object hand to hand, 5.5 months; lifts cup with handle, 5.8 months; looks for fallen spoon, 6.0 months; playful response to mirror, 6.2 months; manipulates bell -interest in detail, 6.5 months; rings bell purposively, 7.8 months; fingers holes in pegboard, 8.9 months. Items failed included: interest in sound production, 5.8 months; attends to scribbling, 5.8 months; vocalizes four different syllables, 7.0 months; attempts to secure three cubes, 7.6 months; listens selectively to familiar words, 7.9 months; says "da-da" or equivalent, 7.9 months.

The child's interest in sound production and his response to sound was slightly below the level of his overall mental development. The plastic barrier does slightly diminish the transmission of sound.

On the Motor Scale, Basal Age was 6.6 whereas Ceiling Age was 7.1 months. Items passed included: rotates wrist, 5.7 months; sits alone thirty seconds or more, 6.0 months; rolls from back to stomach, 6.4 months; sits alone steadily, 6.6 months; sits alone good coordination, 6.9 months; prewalking progession, 7.1 months. Items failed included: scoops pellet, 6.8 months; early stepping movements, 7.4 months; pulls to standing position, 8.1 months; raises self to sitting position, 8.3 months.

The test findings indicated that, on average, he was at least a month advanced in both mental and motor development.

\section{FOLLOW-UP EVALUATION}

The second evaluation, done on September 9, 1974 at age 3 years, 4 days, was made when he was in his isolator at home with his mother present. The Stanford-Binet (50) test items in Table 1 afford a cross-section of his intellectual development.

It was possible to administer only one test item at Year V (Definitions), and he passed this.

The high level of success in the Discrimination of Forms Test at Year Level IV is especially noteworthy. He identified all 10 geometric figures and at times correctly named them. This indicated unusual ability in determining size and shape of objects. This type of perceptual skill could be related to the environment he lives in, where the geometry of all the mechanical aspects of his isolation chamber has an enhanced valence for him and certainly would influence his entire framework of object relations.

His general language skills, measured by the McCarthy Scales (34), were within the 4-year, 6-month to 5-year level. 